\title{
Investigation of the electron capture process in semiclassical plasma
}

Madina M. Seisembayeva, Karlygash N. Dzhumagulova, Tlekkabul S. Ramazanov

\begin{abstract}
In this work, the process of electron capture in partially ionized plasma is considered. Electron-atom interaction was described by the effective interaction potential, which takes into account the screening effect at large distances and the diffraction effect at the small distances. The results of numerical calculations of the electron capture radius, differential cross-section for different values of the coupling and density parameters are presented. The differential cross-section was obtained on the basis of perturbation theory and also by solving of the equation of motion of the projectile electron.
\end{abstract}

Key words: capture time $\cdot$ differential cross-section $\bullet$ partially ionized plasma $\bullet$ perturbation theory $\bullet$ effective interaction potential $\bullet$ electron capture radius

M. M. Seisembayeva, K. N. Dzhumagulova ${ }^{\bowtie}$, T. S. Ramazanov

Institute of Experimental and Theoretical Physics,

Al-Farabi Kazakh National University,

71 al-Farabi Str., Almaty 050040, Kazakhstan,

Tel.: + 7 (707) 739 0195, Fax: +00 (772) 729 24988,

E-mail: dzhumagulova.karlygash@gmail.com

Received: 24 September 2015

Accepted: 16 November 2015

\section{Introduction}

Investigation of the interaction between particles and plasma properties is of great interest in many areas of physics such as atomic and plasma physics. Also, it is important for the development of the plasma technologies. One of the elementary processes in plasma is the electron capture process due to electron and atom collision. The process of the electron capture by an atom was investigated in many studies [1-6]. As an example, one can mention a paper, where an influence of electron-exchange and plasma screening on the electron capture process in degenerate semiconductor quantum plasmas was investigated. The charge capture radius and charge capture cross-section were obtained on the basis of the Shukla-Eliasson effective potential between the electron and ion.

In present paper, electron capture by the hydrogen atom was considered. The neutral hydrogen atom can be transformed into the negative hydrogen ion due to polarization electron capture. The electron capture by hydrogen atom plays an important role in partially ionized plasmas. It is known that the negative hydrogen ion participates in the reaction $\mathrm{H}^{-}$ $+\mathrm{H} \rightarrow \mathrm{H}_{2}+\mathrm{e}^{-}$, it is the one of the main channel for forming $\mathrm{H}_{2}$ molecule. Negative ion beam, pulled out of the hydrogen plasma, is used for cleaning of metal surfaces and creation of the stream of hydrogen atoms in high-energy accelerators and fusion devices.

In work [5], the electron capture cross-section was analyzed on the basis of perturbation theory, 
but authors did not present the analysis of results for different values of plasma parameters. In our study, we calculated the electron capture cross-section on the basis of equations of the electron motion in the field of an atom, and perturbation theory at different values of plasma parameters for a comparative analysis. Indeed, a hyperbolic orbit trajectory is to be used and a comparison with this approach will be a subject of future work.

In the present work, we used the interaction potential between the electron and the atom in partially ionized hydrogen plasmas, which was described in works [7-9]. This effective potential, taking into account the quantum-mechanical effects of diffraction of particles and plasma screening effects, has finite values at the distance close to zero. It has the following form:

$$
\begin{aligned}
\Phi_{e a}(r) & =-\frac{e^{2} \alpha}{2 r^{4}\left(1-4 \frac{\hbar_{e a}^{2}}{r_{d}^{2}}\right)} \\
& \cdot\left(e^{-B r}(1+B r)-e^{-A r}(1+A r)\right)^{2}
\end{aligned}
$$

where $\quad A^{2}=\frac{1}{2 \lambda_{e a}^{2}}\left(1+\sqrt{1-4 \lambda_{e a}^{2} / r_{\mathrm{D}}^{2}}\right)$,

$$
B^{2}=\frac{1}{2 \lambda_{e a}^{2}}\left(1-\sqrt{1-4 \lambda_{e a}^{2} / r_{\mathrm{D}}^{2}}\right)
$$

Here, $\lambda_{e a}=\hbar /\left(2 \pi \mu_{e a} k_{\mathrm{B}} T\right)^{1 / 2}$ is the de Broglie wavelength, $r_{\mathrm{D}}=\sqrt{k_{\mathrm{B}} T /\left(4 \pi n e^{2}\right)}$ is the Debye length, $k_{\mathrm{B}}$ denotes the Boltzmann constant, $\mu_{e a}=m_{\mathrm{e}} m_{a} /\left(m_{\mathrm{e}}\right.$ $\left.+m_{a}\right) \approx m_{\mathrm{e}}$ is the reduced mass of the atom and electron pair, $\alpha$ is the polarizability of the atom. For hydrogen atom, it equals to $4.5 a_{\mathrm{B}}^{3}, a_{\mathrm{B}}=\hbar^{2} /\left(m_{\mathrm{e}} e^{2}\right)$, is the Bohr radius.

For a description of the properties of a partially ionized plasma, it is convenient to use dimensionless parameters characterizing plasma state at certain densities and temperatures. One of such parameters is the coupling parameter:

$$
\Gamma=\frac{\left(Z e^{2}\right)}{a k_{\mathrm{B}} T}
$$

where $T$ is plasma temperature, $a=[3 /(4 \pi n)]^{1 / 3}$ is average distance between particles. The density parameter $r_{s}=a / a_{\mathrm{B}}$.

Applying these coupling and density parameters, the effective potential (1) in dimensionless form looks like this:

$$
\begin{aligned}
& \frac{\Phi_{e a}(R)}{k_{\mathrm{B}} T}=\frac{\left(\alpha / a_{\mathrm{B}}^{3}\right) \Gamma r_{s}}{2 R^{4}\left(1-\frac{12 \Gamma^{2}}{\pi r_{s}}\right)} \\
& \cdot\left(e^{-\bar{B} R}(1+\bar{B} R)-e^{-\bar{A} R}(1+\bar{A} R)\right)^{2} \\
& \bar{A}^{2}=\frac{\pi}{r_{s} \Gamma}\left(1+\sqrt{1-\frac{12 \Gamma^{2}}{\pi r_{s}}}\right) \\
& \bar{B}^{2}=\frac{\pi}{r_{s} \Gamma}\left(1-\sqrt{1-\frac{12 \Gamma^{2}}{\pi r_{s}}}\right)
\end{aligned}
$$

where $R=r / a_{\mathrm{B}}$ is interparticle distance in units of the Bohr radius.

\section{Theory and applied methods}

The Bohr-Lindhard method has been applied to obtain the electron capture radius, capture time, and electron capture probability. In the Bohr-Lindhard method [2], it has been known that the electron capture happens when the distance between the atom and moving electron is smaller than the electron capture radius $r_{\text {cap }}$. This electron capture radius is determined by equating the kinetic energy of moving electron and the interaction energy between the electron and the atom.

$$
\frac{e^{2} \alpha}{2 r_{\text {cap }}^{4}\left(1-4 \frac{\hbar^{2}}{r_{d}^{2}}\right)}\left(\begin{array}{l}
e^{-B r_{\text {cap }}}\left(1+B r_{\text {cap }}\right) \\
-e^{-A r_{\text {cap }}}\left(1+A r_{\text {cap }}\right)
\end{array}\right)^{2}=\frac{1}{2} m v_{p}^{2}
$$

where $v_{p}$ is the velocity of moving electron, $1 / 2 m v_{p}^{2}$ is the kinetic energy of moving electron. The interaction energy provided by the polarization interaction should be greater than the kinetic energy of the moving electron in the frame of the hydrogen atom. The dimensionless form of Eq. (3) is given by

$$
\text { (4) } \frac{\left(\alpha / a_{\mathrm{B}}^{3}\right) \Gamma r_{s}}{2 R_{\text {cap }}^{4}\left(1-\frac{12 \Gamma^{2}}{\pi r_{s}}\right)}\left(\begin{array}{l}
e^{-\bar{B} R_{\text {cap }}}\left(1+\bar{B} R_{\text {cap }}\right) \\
-e^{-\bar{A} R_{\text {cap }}}\left(1+\bar{A} R_{\text {cap }}\right)
\end{array}\right)^{2}-\frac{\bar{v}_{p}^{0}}{2}=0
$$

where $\bar{v}_{p}=v_{p} / v_{t h}, v_{t h}$ is the thermal velocity of electron, $R_{\text {cap }}=r_{\text {cap }} / a_{\mathrm{B}}$ is the dimensionless capture radius.

The time of the capture was determined as the time, when electron moves within circle with capture radius. It was found from the ratio of the traversed path of the electron to the velocity of the electron.

$$
t_{\text {cap }}=\left\{\begin{array}{cl}
\frac{2 \sqrt{r_{\text {cap }}^{2}-b^{2}}}{v_{p}^{2}} & \text { for } b<r_{\text {cap }} \\
0 & \text { for } b \geq r_{\text {cap }}
\end{array}\right.
$$

It should be noted that the electron is captured, if the impact parameter $b$ is less than the capture radius $b<r_{\text {cap }}$. The impact parameter $b$ is the vertical distance between the centers of the moving electron and the atom.

The electron capture probability is defined by the ratio of the collision time to the electron orbital time:

$$
P_{\text {cap }}\left(b, v_{p}\right)=\frac{1}{\tau} \int_{-t_{\text {cap }}}^{t_{\text {cap }}} d t
$$

Using the electron capture probability, the electron capture cross-section was calculated on the basis of the following expression:

$$
\sigma_{\text {cap }}=2 \pi \int d b b P_{\text {cap }}\left(b, v_{p}\right)
$$

where $\tau=a_{n} / v_{n}$ is the electron orbital time, $a_{n}=$ 
$n^{2} a_{\mathrm{B}} / Z$ is the $n$-th Bohr radius of the hydrogenic atom, $Z$ is atomic number of the atom, $v_{n}=Z \alpha c / n$ is the electron velocity of the $n$-th Bohr orbit.

In this work, the pair collision of impacting electron with atom was considered. The influence of other plasma particles is taken into account by the effective potential (1). The equations of motion of the electron in the field of the motionless hydrogen atom were numerically solved. The system has the general form:

$$
\begin{gathered}
\vec{r}(t+\Delta t)=\vec{r}(t)+\vec{v}(t) \Delta t+\frac{\vec{F}(t) \Delta t^{2}}{2 m_{\mathrm{e}}} \\
\vec{v}(t+\Delta t)=\vec{v}(t)+\frac{(\vec{F}(t+\Delta t)+\vec{F}(t)) \Delta t}{2 m_{\mathrm{e}}}
\end{gathered}
$$

Here, $\vec{r}, \vec{v}$ and $m_{\mathrm{e}}$ are the coordinates, velocity, and mass of the electron, $\vec{F}=-\operatorname{grad} \Phi_{e a}(r)$ is the force acting on the electron in the field of the atom. Initial conditions are determined by the impact parameter $b$ and the initial velocities $v_{x}=v_{0}, v_{y}=0$.

\section{Results}

Figure 1 shows the dependence of the electron capture radius on the velocity of the moving electron. It can be seen that with an increase in velocity the capture radius decreases. When the density parameter decreases, the electron capture radius decreases too.

Capture radii for different coupling parameters are shown in Fig. 2. A change of the capture radius with an increase in the coupling parameter is explained by the behavior of the effective potential, that is, in dense plasma the effect of diffraction is significant at small distances, and the effect of screening at large distances.

Figures 3 and 4 show the differential cross-section of electron capture obtained on the basis of the perturbation theory (Eqs. (5)-(7)). These figures represent the dependence of the electron capture cross-section on the impact parameter for various values of the coupling parameter and density param-

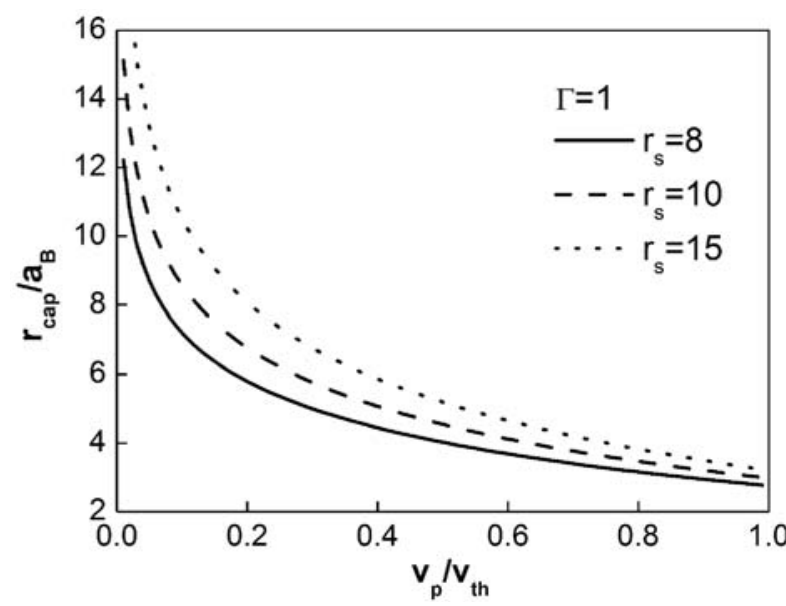

Fig. 1. The electron capture radius as function of the velocity of the moving electron for different values of the density parameter.

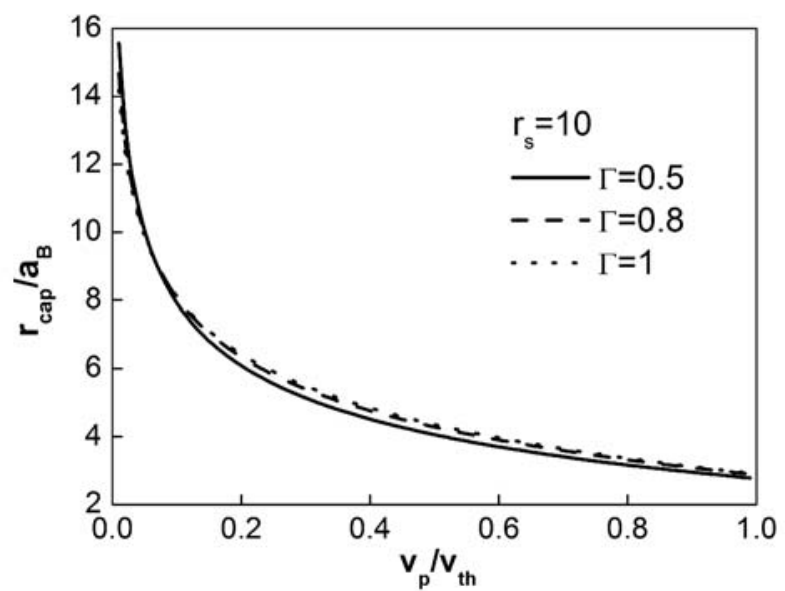

Fig. 2. The electron capture radius as function of the velocity of the moving electron for different values of the coupling parameter.

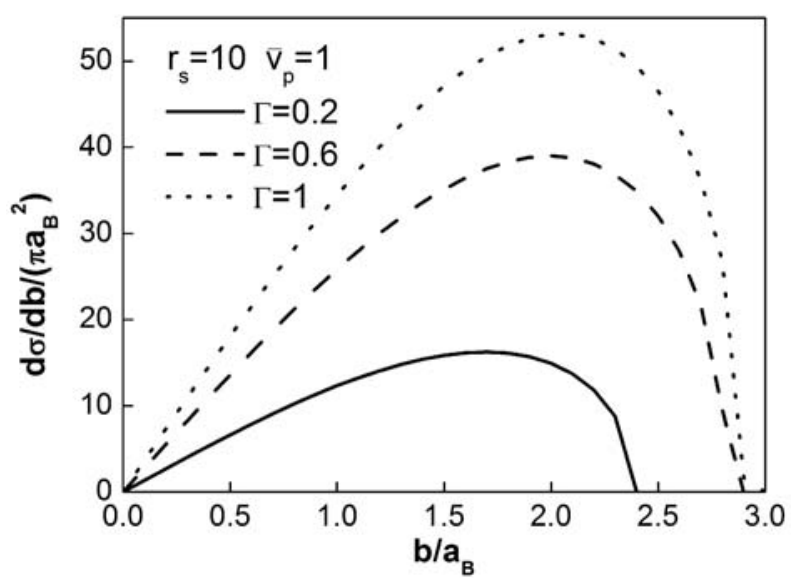

Fig. 3. The differential cross-section computed for different values of the coupling parameter.

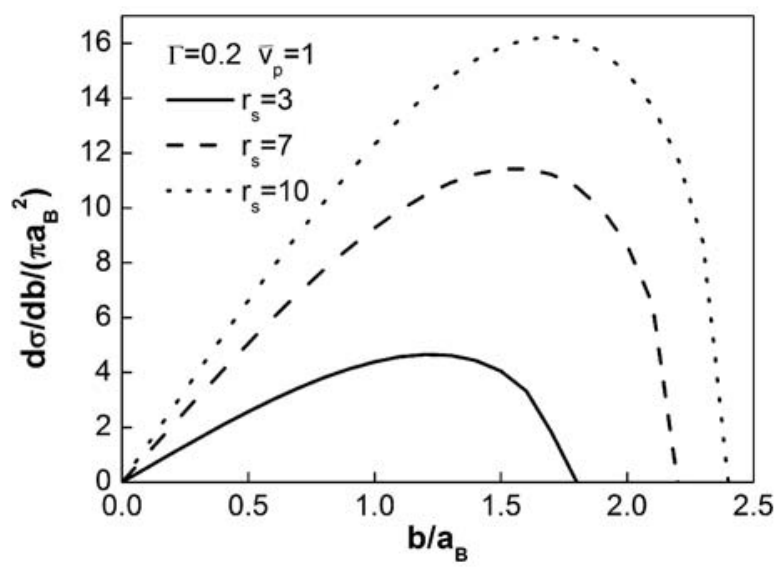

Fig. 4. The differential cross-section computed for different values of the density parameter.

eter. As one can see, the differential cross-section significantly increases with grow of the temperature, and decreases in more dense plasma.

Figure 5 represents the trajectories of the impact electron near atom obtained for the different impact parameters and initial velocity $\bar{v}_{x}=1$ by solving the equations of motion (Eqs. (8), (9)). One can see that the atom attracts electron due to polariza- 


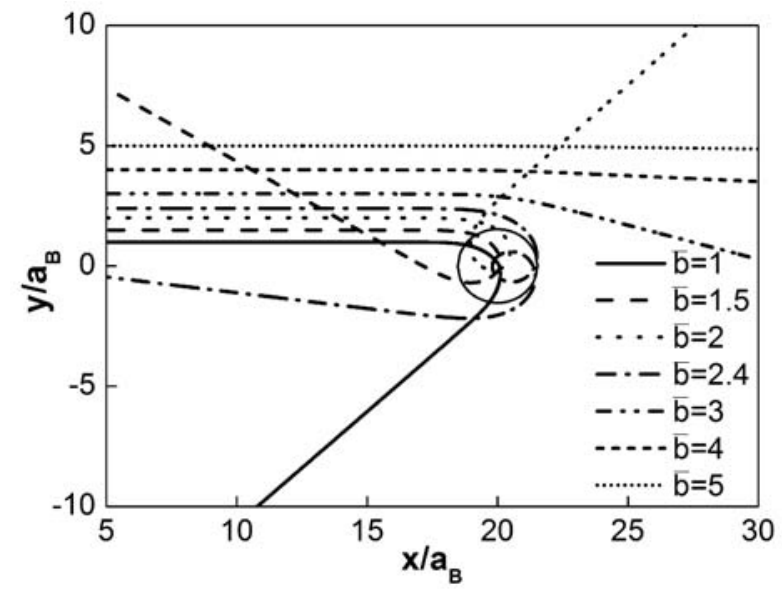

Fig. 5. The trajectories of the electron near the atom calculated by the equation of motion, $G=0.2, r_{s}=3$, $\bar{v}_{x}=1, \bar{v}_{y}=1$.

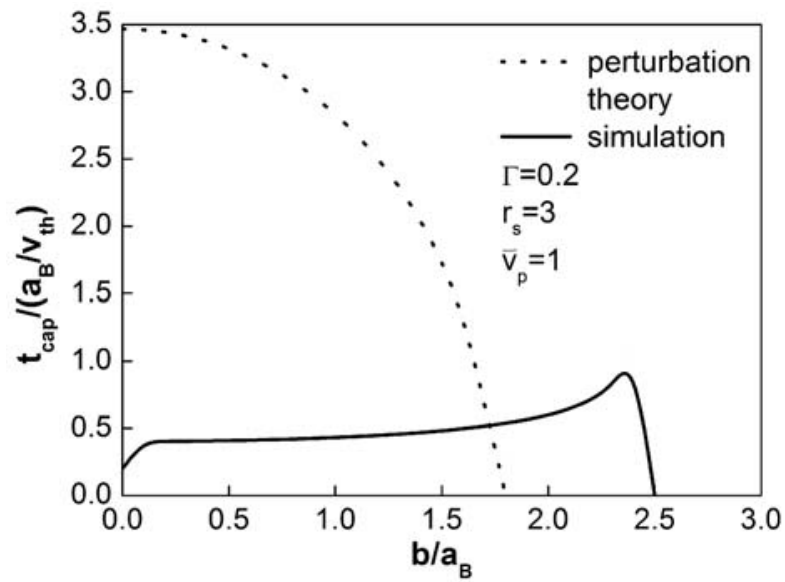

Fig. 6. Comparison of the electron capture times on the basis of the perturbation theory and simulation.

tion interaction and trajectories of the electrons are not straight or simple hyperbolic, they may even be screwed.

A comparison of the electron capture times and differential cross-sections, as obtained by the perturbation theory and by solving the equation of motion, is presented in Figs. 6 and 7.

A trajectory of the moving electron in a framework of the perturbation theory is a straight one.

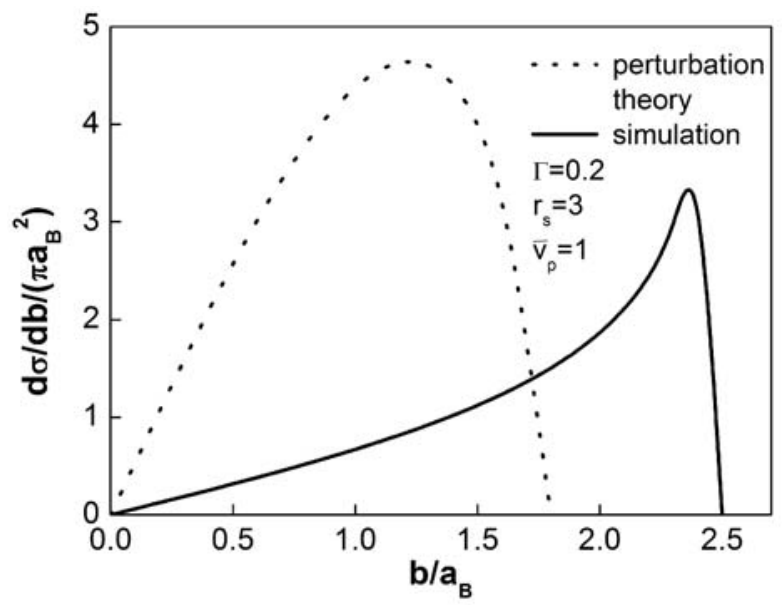

Fig. 7. Comparison of the differential cross-sections obtained on the basis of the perturbation theory and simulation.

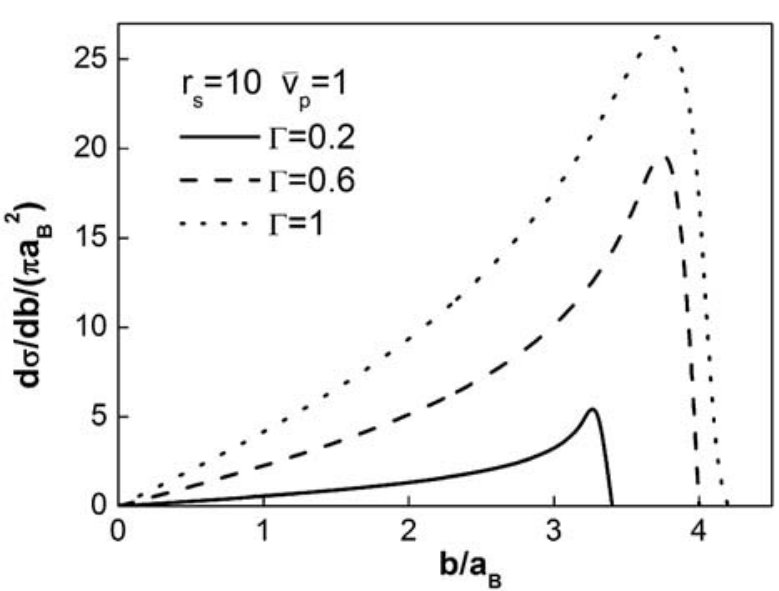

Fig. 8. The differential cross-sections obtained on the basis of simulation for different coupling parameters.

The trajectory of the moving electron, as obtained by solving the equation of motion, is not a straight line. Therefore, the differential cross-section is not equal to zero at impact parameters $\bar{b}>R_{\text {cap }}$. In this case the electron can be captured by an atom even at impact parameters $\bar{b}>R_{\text {cap }}$, if its trajectory goes into the sphere with radius $\bar{R}_{\text {cap }}$. The peak of the differential cross-section in Fig. 7 corresponds to the peak of the capture time in Fig. 6. Besides this, electron spends more time in this sphere with an increase in the impact parameter, e.g., capture time increases. It is interesting result because it flatly contradicts to behavior of a capture time on the basis of the perturbation theory, as shown in Fig. 6 .

Figure 8 represents the differential cross-section of electron capture as a function of the impact parameter for various values of the coupling parameter. This electron capture cross-section was obtained by solving the equation of motion. In this figure, one can see that the electron capture cross-section increases with growing of the coupling parameter, i.e., with a decrease in the temperature.

\section{Conclusion}

In this paper, the process of electron capture by the hydrogen atom was investigated. The interaction between the electron and the atom takes into account the screening effect at large distances and the effect of diffraction at small distances.

The electron capture radius for different values of the coupling parameters and density parameters was calculated. The results showed that the electron capture radius decreases with an increase in the velocity of the electrons. Differential cross-section was obtained on the basis of perturbation theory and the equations of motion. Results were compared. The differential cross-section obtained on the basis of calculated trajectories does not equal to zero at some values of the impact parameter $b>r_{\text {cap }}$ and grows with an increase in the coupling parameter. These results provide useful information on electron capture process in partially ionized plasma. 
Acknowledgment. The authors gratefully acknowledge Professor Y. -D. Jung for useful discussions and warm hospitality while visiting at the Hanyang University.

This work was performed at the al-Farabi Kazakh National University in Almaty, Kazakhstan.

\section{References}

1. Ben-Itzhak, I., Jaint, A., \& Weaver, O. L. (1993). Impact parameter dependence of classical probability from any initial state by fast bare projectiles. J. Phys. $B, 26,1711-1726$.

2. Brandt, D. (1983). A simple classical model for the impact parameter dependence of electron capture. Nucl. Instrum. Methods, 214, 93-96.

3. Jung, Y. -D. (1997). Electron capture from one- and two-electron atoms by fast positrons in dense plasmas. Phys. Plasma, 4(1), 16-20.

4. Jung, Y. -D., \& Akbari-Moghanjoughi, M. (2014). Electron-exchange effects on the charge capture process in degenerate quantum plasmas. Phys. Plasma, 21, 032108-(1-6).
5. Ki, D. -H., \& Jung, Y. -D. (2012). Formation of negative hydrogen ion: Polarization electron capture and nonthermal shielding. J. Chem. Phys., 137(9), 094310-(1-15).

6. Ryufuku, H., \& Watanabe, T. (1979). Total and partial cross sections for charge transfer in collisions of multicharged ions with atomic hydrogen. Phys. Rev. A, 20(5), 1828-1837.

7. Ramazanov, T. S., Dzhumagulova, K. N., \& Akbarov, A. Z. (2006). Cross sections and transport coefficients of dense partially ionized semiclassical plasma. $J$. Phys. A, 39, 4335-4340.

8. Ramazanov, T. S., Dzhumagulova, K. N., \& Gabdullin, M. T. (2006). Microscopic and thermodynamic properties of dense semiclassical partially ionized hydrogen plasma. J. Phys. A, 39, 4469-4474.

9. Ramazanov, T. S., Dzhumagulova, K. N., \& Omarbakiyeva, Y. A. (2005). Effective polarization interaction potential "charge-atom" for partially ionized dense plasma. Phys. Plasma, 12, 092702-(1-4). 